POS PROCEEDINGS

\title{
Current results of the experiments at VEPP-2000
}

M.N.Achasov ${ }^{a b}$, R.R.Akhmetshin ${ }^{a}$, A.V.Anisenkov ${ }^{a}$, V.M.Aulchenko ${ }^{a b}$, V.S.Banzarov $^{a}$, L.M.Barkov ${ }^{a}$, A.Yu.Barnyakov ${ }^{a}$, N.S.Bashtovoy ${ }^{a}$, K.I.Beloborodov $^{a b}$, A.V.Berdyugin $^{a b}$, D.E.Berkaev $^{a b}$, A.G.Bogdanchikov ${ }^{a}$, , A.E.Bondar ${ }^{a b}$, A.A.Botov ${ }^{a}$, A.V.Bragin $^{a}$, T.V.Dimova ${ }^{a}$, V.P.Druzhinin ${ }^{a b}$, S.I.Eidelman ${ }^{a b}$, D.A.Epifanov ${ }^{a f}$, L.B.Epshteyn $^{a c}$, A.L.Erofeev ${ }^{a}$, G.V.Fedotovich ${ }^{a b}$, S.E.Gayazov ${ }^{a b}$, V.B.Golubev $^{a b}$, A.A.Grebenuk ${ }^{a b}$, K.A.Grevtsov ${ }^{a b}$, D.N.Grigoriev ${ }^{a b c}$, E.M.Gromov ${ }^{a}$, F.V.Ignatov ${ }^{a}$, L.V.Kardapoltsev $^{a b}$, S.V.Karpov ${ }^{a}$, V.F.Kazanin ${ }^{a b}$, A.G.Kharlamov ${ }^{a b}$, B.I.Khazin $^{a b}$, A.N.Kirpotin $^{a}$, I.A.Koop ${ }^{a b}$, A.A.Korol ${ }^{a b}$, S.V.Koshuba ${ }^{a}$, O.A.Kovalenko ${ }^{a b}$, D.P.Kovrizhin ${ }^{a}$, A.N.Kozyrev ${ }^{a}$, E.A.Kozyrev ${ }^{a b}$, P.P.Krokovny ${ }^{a b}$, A.S.Kupich ${ }^{a}$, A.E.Kuzmenko ${ }^{a c}$, A.S.Kuzmin ${ }^{a}$, I.B.Logashenko ${ }^{a b}$, P.A.Lukin ${ }^{a b}$, A.P.Lysenko ${ }^{a}$, K.A.Martin $^{a c}$, K.Yu.Mikhailov ${ }^{a b}$, A.E.Obrazovsky ${ }^{a}$, V.S.Okhapkin ${ }^{a}$, E.V.Pakhtusova ${ }^{a}$, E.A.Perevedentsev ${ }^{a}$, Yu.N.Pestov ${ }^{a}$, A.S.Popov ${ }^{a}$, Yu.S.Popov ${ }^{a}$, G.P.Razuvaev ${ }^{a b}$, Yu.A.Rogovsky ${ }^{a}$, A.L.Romanov ${ }^{a}$, A.A.Ruban ${ }^{a}$, N.M.Ryskulov ${ }^{a}$, A.E.Ryzhenenkov ${ }^{a b}$, S.I.Serednyakov ${ }^{a b}$, P.Yu.Shatunov ${ }^{a}$, Yu.M.Shatunov ${ }^{a}$, V.E.Shebalin ${ }^{a}$, D.N.Shemyakin $^{a b}{ }$ D.A.Shtol ${ }^{a}$, B.A.Shwartz ${ }^{* a}$, D.B.Shwartz $^{a b}$, A.L.Sibidanov $^{a d}$, Z.K.Silagadze $^{a b}$, E.P.Solodov ${ }^{a b}$, I.K.Surin ${ }^{a}$, A.A.Talyshev ${ }^{a b}$, V.M.Titov $^{a}$, A.V.Vasiljev ${ }^{a}$, A.I.Vorobiov $^{a}$, Yu.V.Yudin ${ }^{a}$, I.M.Zemlyansky ${ }^{a}$, Yu.M.Zharinov ${ }^{a}$

${ }^{a}$ Budker Institute of Nuclear Physics, SB RAS, Novosibirsk, 630090, Russia

${ }^{b}$ Novosibirsk State University, Novosibirsk, 630090, Russia

${ }^{c}$ Novosibirsk State Technical University, Novosibirsk, 630092, Russia

${ }^{d}$ University of Sydney, Falkiner High Energy Physics, NSW 2006, Sydney, Australia

${ }^{e}$ Brookhaven National Laboratory, P.O. Box 5000 Upton, NY 11973-5000, USA

${ }^{f}$ University of Tokyo, Department of Physics, 7-3-1 Hongo Bunkyo-ku Tokyo, 113-0033, Japan

E-mail: shwartz@inp.nsk.su

Experiments at BINP $e^{+} e^{-}$collider VEPP-2000 with two detectors, CMD-3 and SND are conducted since December 2010. The energy range comprized by this collider is from 0.3 to $2 \mathrm{GeV}$ and the project luminosity up to $1 \cdot 10^{32} \mathrm{~cm}^{-2} \mathrm{~s}^{-1}$. By now the peak luminosity exceeding $1 \cdot 10^{31} \mathrm{~cm}^{-2} \mathrm{~s}^{-1}$ was achieved and each detector collected about $60 \mathrm{pb}^{-1}$ of integrated luminosity. Preliminary results of the experiments are reviewed in this report.

XV International Conference on Hadron Spectroscopy-Hadron 2013

4-8 November 2013

Nara, Japan

${ }^{*}$ Speaker. 


\section{Introduction}

Although a study of $e^{+} e^{-}$annihilation into hadrons at low energies has a long history, new precise measurements are still highly desired since these provide important information about interactions of light quarks and spectroscopy of their bound states.

One of the important subjects in this field is a precise determination of the total hadron production cross section characterized by the ratio $R$ :

$$
R(s)=\frac{\sigma\left(e^{+} e^{-} \rightarrow \text { hadrons }\right)}{\sigma\left(e^{+} e^{-} \rightarrow \mu^{+} \mu^{-}\right)}, \quad \sigma_{\mu^{+} \mu^{-}}=\frac{4 \pi \alpha^{2}}{3 s}=\frac{86.85 \mathrm{nb}}{s\left[\mathrm{GeV}^{2}\right]} .
$$

This ratio is closely connected to the muon anomalous magnetic moment calculation as well as running electromagnetic and strong interaction constants.

The former issue became especially interesting in the last years, after high precision measurements of the muon anomalous magnetic moment, $a_{\mu}$, performed at Brookhaven National Laboratory [1]. This experiment achieved a relative accuracy in $a_{\mu}$ value of $5 \cdot 10^{-7}: a_{\mu}=$ $(11659208.9 \pm 6.3) \cdot 10^{-10}$.

The accuracy of the theoretical calculations via the Standard Model is dominated at present by the precision of the hadronic contribution [2,3]. At present the calculations of $a_{\mu}$ based on the data from $e^{+} e^{-}$colliders result in a difference between theoretical and experimental values of more than 3 standard deviations (see for review [4]). One of the latest works [5] claims even larger discrepancy (about $4 \sigma$ ). If such a difference would be really proved, that could be a direct indication of New Physics.

At present two new projects for muon (g-2) measurements, at FNAL in USA and J-PARC in Japan, are under development and construction [6,7]. An improvement of the accuracy to about $2 \cdot 10^{-10}$ is expected in these projects. Then, the accuracy of the hadronic contribution becomes a crucial issue.

The VEPP-2M collider [8] with the luminosity of $3 \times 10^{30} \mathrm{~cm}^{-2} \mathrm{~s}^{-1}$ was the main supplier of the precise data on the hadronic cross sections in the energy range up to $1.4 \mathrm{GeV}$ for more than 25 years. The latest cycle of experiments from 1992 to 2000, performed at this collider by two detectors, the CMD-2 [9] and the SND [10], provided the most accurate data on $e^{+} e^{-}$annihilation into hadrons in the energy range $0.36<\sqrt{s}<1.4 \mathrm{GeV}$.

Since December 2010 experiments at the new VEPP-2000 collider are going on. This talk discusses the current status and results of these experiments.

\section{The VEPP-2000 collider and detectors}

This collider has an energy range from 0.3 to $2 \mathrm{GeV}$ at the center-of-mass which is wider than that of VEPP-2M while the project luminosity is up to $10^{32} \mathrm{~cm}^{-2} \mathrm{~s}^{-1}$ [11]. The schematic view of the collider as well as its main parameters are presented in Fig. 1 in comparison to the VEPP-2M. To achieve high luminosity a special magnetic structure of the ring is used to realize the concept of Round Beams (RB) developed at the BINP. Two focussing solenoids with $12 \mathrm{~T}$ magnetic field are installed at each interaction region. The collider operates with one electron and one positron bunches containing up to $10^{11}$ particles. 


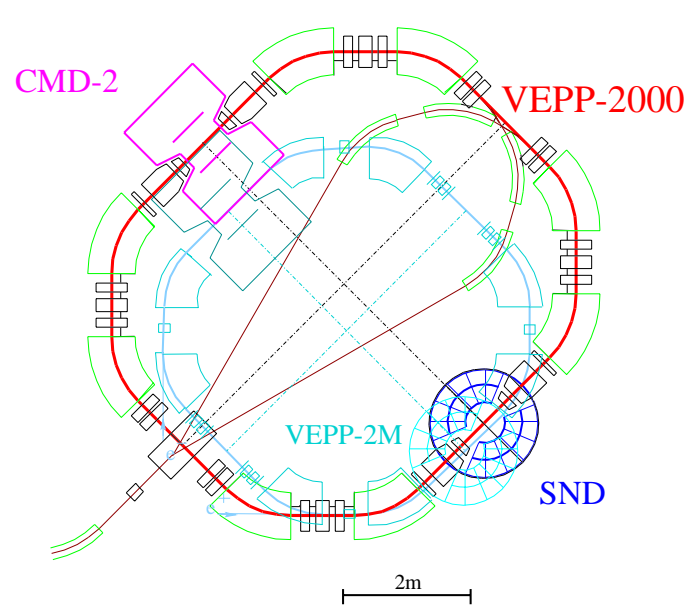

VEPP-2M \& VEPP-2000 parameters

\begin{tabular}{|l|c|c|c|}
\hline & VEPP-2M & \multicolumn{2}{|c|}{ VEPP-2000 } \\
\hline $\mathbf{E}(\mathbf{M e V})$ & $\mathbf{5 1 0}$ & 510 & 900 \\
\hline $\boldsymbol{\Pi}(\mathbf{c m})$ & $\mathbf{1 7 8 8}$ & 2235 & 2235 \\
\hline $\mathcal{I}^{+}, \mathcal{I}^{-}$ & 40 & 34 & 200 \\
$(\mathbf{m A})$ & & & \\
\hline$\varepsilon \cdot \mathbf{1 0}^{\mathbf{5}}$ & $\mathbf{3}$ & 0.5 & 1.6 \\
$(\mathbf{c m} \cdot \mathbf{r a d})$ & & & \\
\hline$\beta_{\mathbf{x}}(\mathbf{c m})$ & 40 & 6.3 & 6.3 \\
$\beta_{\mathbf{z}}(\mathbf{c m})$ & $\mathbf{5}$ & 6.3 & 6.3 \\
\hline$\xi_{\mathbf{x}}$ & $\mathbf{0 . 0 1 6}$ & 0.075 & 0.075 \\
$\xi_{\mathbf{z}}$ & $\mathbf{0 . 0 5 0}$ & 0.075 & 0.075 \\
\hline $\mathcal{L}\left(\mathbf{c m}^{-2} \mathbf{s}^{-1}\right)$ & $\mathbf{3} \cdot 10^{30}$ & $\mathbf{1} \cdot 10^{31}$ & $1 \cdot 10^{32}$ \\
\hline
\end{tabular}

Figure 1: New collider VEPP-2000 $\left(\sqrt{s_{\max }}=2 \mathrm{GeV}\right)$ in comparison to VEPP-2M $\left(\sqrt{s_{\max }}=1.4 \mathrm{GeV}\right)$. Parameters of the VEPP-2M collider at the beam energy of $510 \mathrm{MeV}$ and VEPP-2000 collider at 510 and $900 \mathrm{MeV}$ are listed in the $2 \mathrm{nd}, 3 \mathrm{~d}$ and 4 th columns of the table respectively.

To measure the beam energy special system based on the Compton back scattering of the laser photons is used [12]. The accuracy of these measurements is $30-50 \mathrm{keV}$.

Maximum luminosity, $2 \cdot 10^{31} \mathrm{~cm}^{-2} \mathrm{~s}^{-1}$, was achieved at the energy $\sqrt{s}=1.6-1.7 \mathrm{GeV}$. The luminosity was limited by a deficit of positrons and maximum energy of the booster ( $825 \mathrm{MeV}$ now) at higher energy. However, the new injection complex based on the $500 \mathrm{MeV}$ linac and cooler ring will start operation in 2014 and has to solve this problem. The booster is upgraded as well to increase the maximum beam energy.

The collider has two interaction regions occupied by two detectors - the CMD-3 and upgraded SND [13].

The CMD-3, general purpose detector constructed on the base of CMD-2, retained the basic structure and some of subdetectors of the latter one. In comparison to previous detector the CMD-3 has following advantages:

- a new drift chamber with twice better resolution that provides much better tracking;

- The LXe barrel calorimeter ( $5.4 X_{0}$ of thickness) placed in front of CsI calorimeter $\left(8.1 X_{0}\right)$ which gives:

- better energy resolution for photons due to thicker detecting material (LXe + CsI);

- much better spatial resolution for photons;

- measurement of the shower profile which improves the $e / \pi$ separation

The main part of the Spherical Neutral Detector (SND) is a finely segmented three layer calorimeter with $560 \mathrm{NaI}(\mathrm{Tl})$ scintillation crystals comprising $90 \%$ of the total solid angle. The upgraded SND detector acquired a particle identification system with threshold aerogel counters and a new drift chamber providing much better tracking. The particle identification system provides $e / \pi$ separation for the particle energy below $450 \mathrm{MeV}$ and $\pi / K$ identification over the whole available energy range. 


\section{Experiments and current results}

During experiments in 2011-2013 several energy scans were done in the energy range $0.32 \mathrm{GeV}<$ $\sqrt{s}<2 \mathrm{GeV}$. Each detector collected about $60 \mathrm{pb}^{-1}$ of integrated luminosity including $34.5 \mathrm{pb}^{-1}$ above the $\phi$-meson mass, 8.3 and $8.4 \mathrm{pb}^{-1}$ at $\omega$ and $\phi$ resonanses, respectively, and $9.4 \mathrm{pb}^{-1}$ in a scan below $\phi$-meson.

First results were obtained for many hadronic final states: $e^{+} e^{-} \rightarrow p \bar{p}, n \bar{n}, \pi^{+} \pi^{-} \pi^{0}, \pi^{+} \pi^{-} \pi^{+} \pi^{-}$, $\pi^{+} \pi^{-} \pi^{0} \pi^{0}, \pi^{+} \pi^{-} \pi^{+} \pi^{-} \pi^{0}, 3\left(\pi^{+} \pi^{-}\right), 2\left(\pi^{+} \pi^{-} \pi^{0}\right), \pi^{+} \pi^{-} 4 \pi^{0}, \eta \pi^{+} \pi^{-}, K_{S} K_{L}, \omega \pi^{0} \rightarrow \pi^{0} \pi^{0} \gamma$ $[14,15,16]$. QED processes $e^{+} e^{-} \rightarrow e^{+} e^{-}$and $\gamma \gamma$ were used to determine the integrated luminosity. Let us consider several of these processes.

\subsection{Pion formfactor measurement}

Since the largest part of the total hadronic contribution to the muon (g-2) comes from the $e^{+} e^{-} \rightarrow \pi^{+} \pi^{-}$channel, precise measurement of its cross section is extremely important. Due to good angular and momentum resolution at the CMD-3 a sample of events with two collinear charged particles, $e^{+} e^{-}, \mu^{+} \mu^{-}, \pi^{+} \pi^{-}$, practically does not contain a background contamination from the other physical processes. A separation of the three two-body processes are based on the particles momenta and the energy deposition in the calorimeter. The former method is effective at the CMS energy below $650 \mathrm{MeV}$ while the latter method can be used above approximately $500 \mathrm{MeV}$. The range of overlapping provides a cross-check of the separation procedures. Very preliminary results of the pion form factor measurements are shown in the Fig. 2 (left plot). An important test of this analysis is a comparison of the number of $e^{+} e^{-} \rightarrow \mu^{+} \mu^{-}$events with that calculated from QED. Fig. 2 (right plot) demonstrates a consistency of these values within $0.5 \%$.
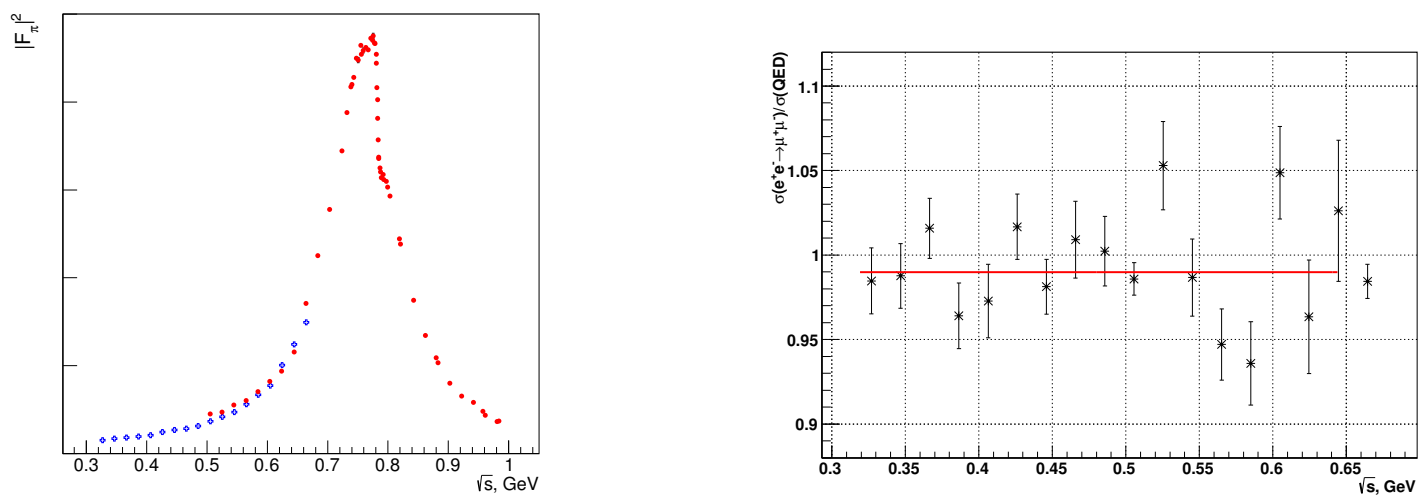

Figure 2: Preliminary results on $\left|F_{\pi}\right|^{2}$ from CMD-3 (left plot). Filled circles - separation done from the calorimeter information, open crosses - from particle momentum. Comparison of the observed number of muon pairs with QED prediction (right plot).

\subsection{Multi-photon channels}

The SND detector is very well suited to multi-photon events detection.

A cross section of the process $e^{+} e^{-} \rightarrow \omega \pi^{0} \rightarrow \pi^{0} \pi^{0} \gamma$, measured by the SND [17] on the base of $30 \mathrm{pb}^{-1}$ of the integrated luminosity, is presented in the Fig. 3. The systematic error of these 


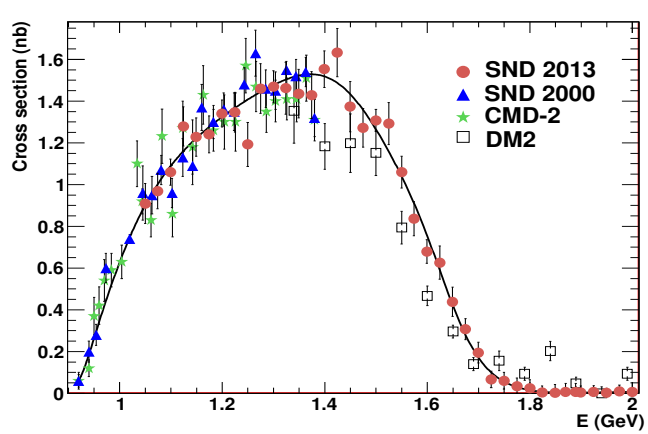

Figure 3: The $e^{+} e^{-} \rightarrow \omega \pi^{0} \rightarrow \pi^{0} \pi^{0} \gamma$ cross section measurement. The curve corresponds to a fit with $\rho(770), \rho^{\prime}(1450)$ and $\rho^{\prime \prime}(1700)$.

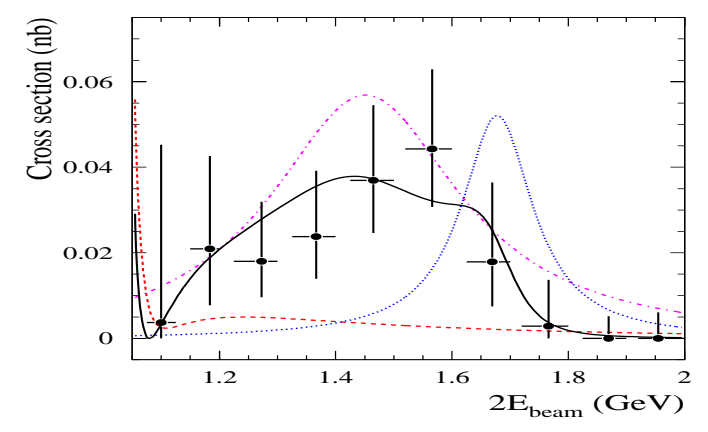

Figure 4: The $e^{+} e^{-} \rightarrow \eta \gamma \rightarrow 7 \gamma$ cross section measurement. Solid curve is obtained by the fit with $\rho, \omega, \phi, \rho^{\prime}(1450)$ and $\phi^{\prime}(1680)$ resonances. Other curves present partial contributions.

measurements is $3.4 \%$. At present it is the most accurate measurement of this cross section between 1.4 and 2.0 GeV. SND results agree with the previous data from the SND [18] and CMD-2 [19] experiments below $1.4 \mathrm{GeV}$ but significantly differ from the DM2 results [20] in the energy range 1.3-2.0 GeV. The measured cross section is well described by the VMD model with three states: $\rho(770), \rho^{\prime}(1450)$ and $\rho^{\prime \prime}(1700)$.

Using $32 \mathrm{pb}^{-1}$ of the integrated luminosity the SND collaboration observed 60 events of the process $e^{+} e^{-} \rightarrow \eta \gamma \rightarrow 7 \gamma$ at the energy above $1 \mathrm{GeV}$ [21]. Results shown in Fig. 4 present the first measurement of this process above $1.4 \mathrm{GeV}$. Detail discussion of this experiment is given in the talk of D.Shtol at this conference.

$3.3 e^{+} e^{-} \rightarrow 3\left(\pi^{+} \pi^{-}\right)$and $e^{+} e^{-} \rightarrow K^{+} K^{-} \pi^{+} \pi^{-}$

Another interesting process, $e^{+} e^{-} \rightarrow 3\left(\pi^{+} \pi^{-}\right)$was measured by CMD-3 collaboration [22]. Long time ago the DM2 collaboration found an evidence of the sharp dip in the energy range near $p \bar{p}$ production threshold [23]. Recently this was confirmed by the BaBar [24]. Results on this channel recently obtained by the CMD-3 are shown in Fig. 5. The results are in a good agreement with the [24]. It worth noting that the energy resolution in the conventional $e^{+} e^{-}$experiment is much better than that in the ISR approach. Thus, the sharp deep can be studied in more detail at VEPP-2000.

Processes with kaons in the final state provide a considerable contribution to the total hadronic cross section. Fig. 6 shows the preliminary CMD-3 results on the $e^{+} e^{-} \rightarrow K^{+} K^{-} \pi^{+} \pi^{-}$cross section in comparison with other experiments. CMD-3 results are in a good agreement with the BaBar data while DM2 measurements are systematically lower. This study is presented in detail by D.Shemyakin at this conference.

\subsection{Nucleons production near threshold}

An interest to the proton pair production near threshold is induced by the specific behaviour of the $p \bar{p}$ cross section in this energy range which was revealed by the previous experiments (see [25] and references therein). Another important value is the $\left|G_{E} / G_{M}\right|$. Results of two groups measured 


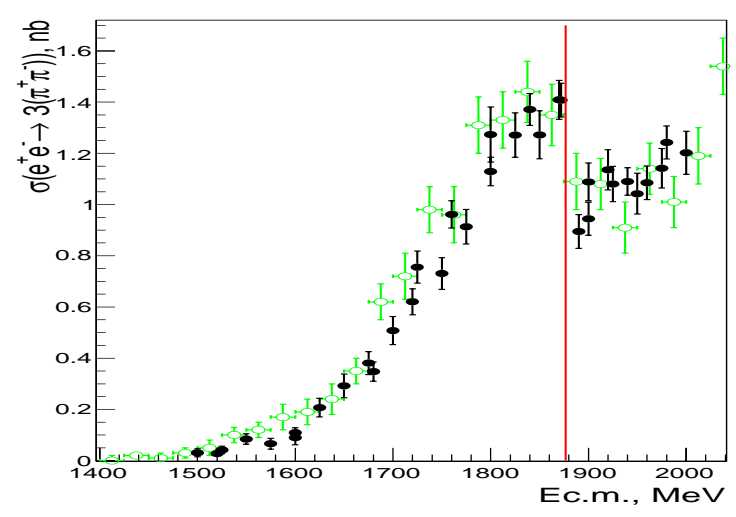

Figure 5: Preliminary results of the measurement of the $e^{+} e^{-} \rightarrow 3\left(\pi^{+} \pi^{-}\right)$cross section obtained by the CMD-3 experiment (black points) in comparison with the BaBar data (green points).

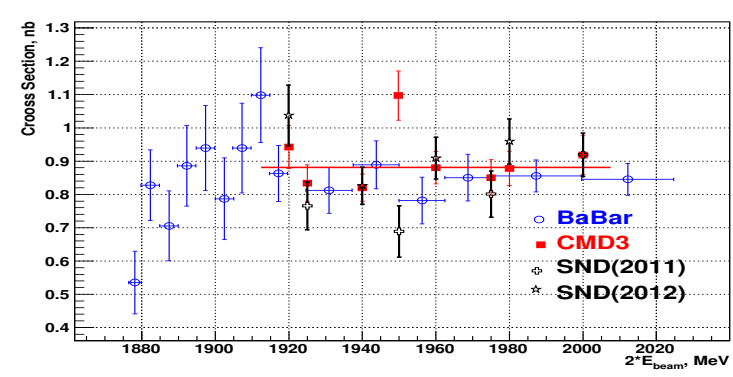

Figure 7: The $e^{+} e^{-} \rightarrow p \bar{p}$ cross section from CMD-3 and SND

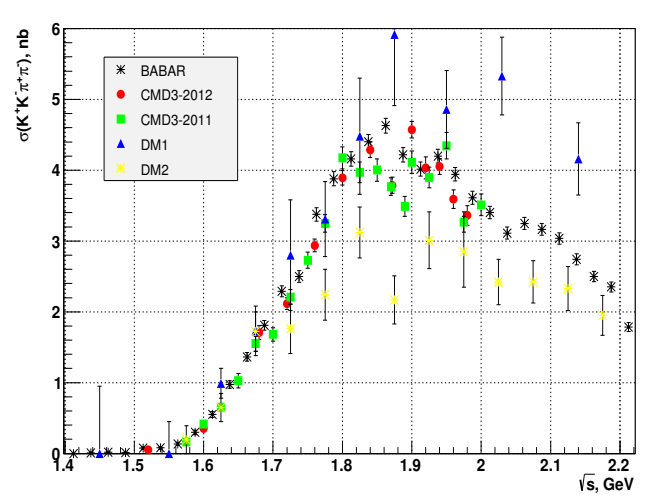

Figure 6: Preliminary result on the $e^{+} e^{-} \rightarrow$ $K^{+} K^{-} \pi^{+} \pi^{-}$cross section with CMD-3.

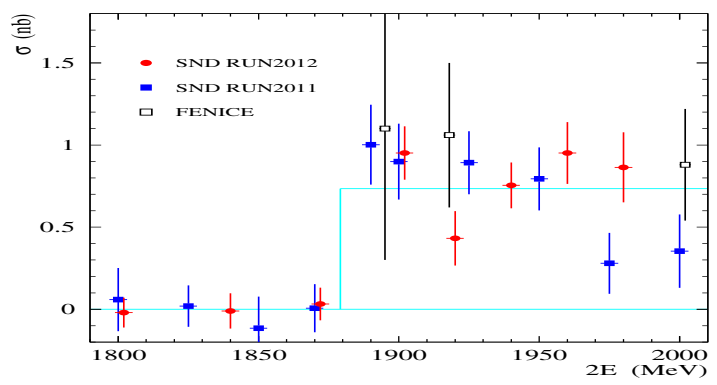

Figure 8: The $e^{+} e^{-} \rightarrow n \bar{n}$ cross section from SND

this value demonstrate strong contradiction [25, 26]. Preliminary results on the process $e^{+} e^{-} \rightarrow p \bar{p}$ in the energy range from the threshold to $2 \mathrm{GeV}$ obtained by the SND and CMD-3 are shown in the Fig. 7 in comparison with the BaBar results. As seen from the figure the results from both detectors are in good agreement with previous experiments. The $\left|G_{E} / G_{M}\right|$ can be evaluated from the angular distribution of the $p \bar{p}$ events which will be performed when higher statistics of data (about $1 \mathrm{fb}^{-1}$ ) will be available. The SND detector obtained first results on the $e^{+} e^{-} \rightarrow n \bar{n}$ as well presented in the Fig. 8.

\section{Conclusion}

Thus, new accelerator principles put on the base of the VEPP-2000 collider were successfully proved. Three years of experiments with the new collider generated considerable amount of data which are analyzed now. At present we have 1.5 year shutdown started last July. This time is intended for booster upgrade to $1 \mathrm{GeV}$ energy as well as commission the new injection complex which will produce enough positrons to reach the project luminosity, $1 \cdot 10^{32} \mathrm{~cm}^{-2} \mathrm{~s}^{-1}$. 
Hopefully, in the next 5-10 years the VEPP-2000 will produce the integrated luminosity of about $1 \mathrm{fb}^{-1}$ which should provide new precise interesting results on the hadron production in $e^{+} e^{-}$annihilation.

We are grateful to the VEPP-2000 team for the excellent machine operation. This work is supported in part by the RFBR grants 11-02-00112-a, 11-02-00328-a, 12-02-00065-a, 12-02-01032-a, 12-02-01250-a. 12-02-31498-mol-a, 12-02-31499-mol-a, 12-02-31501-mol-a, 13-02-00215-a.

\section{References}

[1] G.W. Bennett et al., Phys. Rev. D73 (2006) 072003.

[2] C. Bouchiat and L. Michel, J. Phys. Radium 22 (1961) 121.

[3] M. Gourdin and E. de Rafael, Nucl. Phys. B10 (1969) 667.

[4] S. Eidelman, Nucl. Phys. B (Proc.Suppl.), 189 (2009) 208.

[5] T. Teubner et al., arXiv:1001.5401 (2010).

[6] Lee Roberts, Nucl. Phys. D - Proc. Suppl., 218 (2011) 237.

[7] T. Mibe, Nucl. Phys. D - Proc. Suppl., 218 (2011) 242.

[8] G.M. Tumaikin, Proc. 10th Int. Conf. on High Energy Part. Accel. (Serpukhov, 1977) Vol.1, p.443. V.V. Anashin et al., Preprint Budker INP 84-114, Novosibirsk, 1984.

Yu.M. Shatunov et al., Proc. 7th European Particle Accelerators Conference EPAC2000, (Vienna, Austria 2000) p.439.

[9] E.V. Anashkin et al., Instrum. Exp. Tech., 49 (2006) 798.

[10] M.N. Achasov et al., Nucl. Instr. Meth. A449 (2000) 125.

[11] A.N. Skrinsky, Nucl. Instr. Meth. A598 (2009) 1.

D. Berkaev et al., ICFA Beam Dyn. Newslett. 48 (2009) 235.

[12] E.V. Abakumova et al., Phys. Rev. Lett., 110 (2013) 140402.

[13] B.I. Khazin, Nucl. Instr. Meth. A623 (2010) 353.

[14] M.N. Achasov et al., Progr. in Part. and Nucl. Phys., 67, (2012) Pages 594.

[15] E.V. Abakumova et al., Preprint BINP 2012-020 (2012) Novosibirsk.

[16] R.R. Akhmetshin et al., EPJ Web of Conferences, 37 (2012) 01009.

[17] M.N. Achasov et al., Phys. Rev. D88 (2013) 054013.

[18] M. N. Achasov et al., Phys. Lett. B 486 (2000) 29

[19] R. R. Akhmetshin et al., Phys. Lett. B 562 (2003) 173

[20] D. Bisello et al., Nucl. Phys. Proc. Suppl. 21 (1991) 111

[21] M.N. Achasov et al., arXiv:1312.7078 [hep-ex].

[22] R.R. Akhmetshin et al., Phys. Lett. B723 (2013) 82.

[23] R. Baldini et al., reported at the "Fenice" workshop, Frascati (1988).

[24] B. Auber et al., (BaBar Collaboration), Phys.Rev.D73, 052003 (2006).

[25] B. Aubert et al., Phys. Rev. D 73, 012005 (2006).

[26] G. Bardin et al., Nucl. Phys. B411, (1994) 3. 